\title{
Inflaton field governed universe from NKK theory of gravity: stochastic approach.
}

\author{
1,3 Mariano Anabitarte* ${ }^{2}$ José Edgar Madriz Aguilar ${ }^{\dagger}$ and ${ }^{1,3}$ Mauricio Bellini ${ }^{\ddagger}$ \\ ${ }^{1}$ Departamento de Física, Facultad de Ciencias Exactas y Naturales, Universidad Nacional de \\ Mar del Plata, Funes 3350, (7600) Mar del Plata, Argentina. \\ ${ }^{2}$ Instituto de Física y Matemáticas, AP: 2-82, (58040) Universidad Michoacana de San Nicolás \\ de Hidalgo, Morelia, Michoacán, México. \\ ${ }^{3}$ Consejo Nacional de Ciencia y Tecnología (CONICET).
}

\begin{abstract}
We study a nonperturbative single field (inflaton) governed cosmological model from a 5D Noncompact Kaluza-Klein (NKK) theory of gravity. The inflaton field fluctuations are estimated for different epochs of the evolution of the universe. We conclude that the inflaton field has been sliding down its (quadratic) potential hill along all the evolution of the universe and a mass of the order of the Hubble parameter. In the model here developed the only free parameter is the Hubble parameter, which could be reconstructed in future from Super Nova Acceleration Probe (SNAP) data.
\end{abstract}

Pacs numbers: 04.20.Jb, 11.10.kk, 98.80.Cq

\section{INTRODUCTION}

The possibility that space-time had more than four dimensions has widely been studied regarding its cosmological aspects since long ago [1]. Investigations have focused on attempts to explain why the universe presently appears to have only four space-time dimensions if it is, in fact, a dynamically evolving $(4+k)$-dimensional manifold ( $k$ being the number of extra dimensions). It has been shown that solutions to the $(4+k)$-dimensional Einstein equations exist, for which 4D space-time expands while the extra dimensions contract or remain constant. It has been also suggested that experimental detection of the time variation of the fundamental constants could provide strong evidence for the existence of extra dimensions

\footnotetext{
*E-mail address: anabitar@mdp.edu.ar

$\dagger$ E-mail address: edgar@itzel.ifm.umich.mx

†E-mail address: mbellini@mdp.edu.ar
} 
[2]. In the past years, there has been a marked resurgence of interest in models with noncompact or large extra-dimensions. Three examples of such scenarios are the most known - namely the braneworld models of Randall and Sundrum (RS) [3] and Arkani-Hamed, Dimopoulos and Dvali (ADD) [4,5], as well as the older Space-Time-Matter theory (STM) [6]. The RS model is motivated from certain ideas in string theory, which suggest that the particles and fields of the standard model are naturally confined to a lower-dimensional hypersurface living in a non-compact, higher-dimensional bulk manifold. The driving goal behind the ADD picture is to explain the discrepancy in scale between the observed strength of the gravitational interaction and the other fundamental forces. This is accomplished by noting that in generic higher-dimensional models with compact extra dimensions, the bulk Newton's constant is related to the effective $4 \mathrm{D}$ constant by factors depending on the size and number of the extra dimensions. Finally, STM or induced matter theory proposes that our universe is an embedded $4 \mathrm{D}$-surface in a vacuum $5 \mathrm{D}$-manifold. In this picture, what we perceive to be the source in the 4D Einstein field equations is really just an artifact of the embedding; or in other words, conventional matter is induced from higher-dimensional geometry.

This paper is devoted to the study of a nonperturbative single field (inflaton) governed cosmological model from a 5D NKK theory of gravity. In a cosmological context, the energy of scalar fields has been argued to contribute to the expansion of the universe [7], and has been proposed to explain inflation as well as the presently accelerated expansion. We have in mind an universe which initially suffers an inflationary expansion that after inflation has a change of phase towards a decelerated expansion (radiation and matter dominated expansions), and thereinafter evolves towards the present day observed accelerated (quintessential) expansion. We consider that the universe is in apparent vacuum on the 5D globally flat $\left(R_{B C D}^{A}=0\right)$ metric. The $5 \mathrm{D}$ apparent vacuum is considered as a purely kinetic Lagrangian for a scalar field minimally coupled to gravity on a $5 \mathrm{D}$ globally flat metric.

\section{REVIEWED AND EXTENDED FORMALISM}

\section{A. The inflaton field in a $5 \mathrm{D}$ vacuum state}

We consider the canonical 5D metric [8]

$$
d S^{2}=\theta\left(\psi^{2} d N^{2}-\psi^{2} e^{2 N} d r^{2}-d \psi^{2}\right),
$$

where $d r^{2}=d x^{2}+d y^{2}+d z^{2}$. Here, the coordinates $(N, \vec{r})$ are dimensionless, the fifth coordinate $\psi$ has spatial units and $\theta$ is a dimensionless parameter that can take the values $\theta= \pm 1$. The metric (1) describes a flat $5 \mathrm{D}$ manifold in apparent vacuum $\left(G_{A B}=0\right)$. Notice we are considering a diagonal metric because we are dealing only with gravitational effects, which are the important ones in the global evolution for the universe. Furthermore, the metric (1) is considered as 3D spatially isotropic and flat: $\frac{r^{2}}{3}=x^{2}=y^{2}=z^{2}$ and globally flat $\left(R_{B C D}^{A}=0\right)$.

To describe the $5 \mathrm{D}$ vacuum universe, we consider an action

$$
I=-\int d^{4} x d \psi \sqrt{\left|\frac{(5) g}{\mid(5)} g_{0}\right|}\left[\frac{{ }^{(5)} R}{16 \pi G}+{ }^{(5)} \mathcal{L}(\varphi, \varphi, A)\right],
$$


for a scalar field $\varphi$, which is minimally coupled to gravity. For the metric $(1),\left|{ }^{(5)} g\right|=$ $\psi^{8} e^{6 N}$ is the absolute value for the determinant of $g_{A B}$ and $\left|{ }^{(5)} g_{0}\right|=\psi_{0}^{8} e^{6 N_{0}}$ is a constant of dimensionalization determined by $\left.\right|^{(5)} g \mid$ evaluated at $\psi=\psi_{0}$ and $N=N_{0}$. Furthermore, ${ }^{(5)} R$ is the $5 \mathrm{D}$ Ricci scalar, $G=M_{p}^{-2}$ is the gravitational constant and $M_{p}=1.210^{19} \mathrm{GeV}$ is the Planckian mass. In this work we shall consider $N_{0}=0$, so that $\left|{ }^{(5)} g_{0}\right|=\psi_{0}^{8}$. Here, the index "0" denotes the value at the end of inflation (i.e., when $\ddot{b}=0$ ). Since we are aimed to describe a manifold in apparent vacuum the Lagrangian density $\mathcal{L}$ in $(2)$ should be only kinetic in origin

$$
{ }^{(5)} \mathcal{L}(\varphi, \varphi, A)=\frac{1}{2} g^{A B} \varphi, A \varphi, B,
$$

where the diagonal tensor metric $g^{A B}$ is given by the line element (1).

Since $\frac{\partial N}{\partial \psi}$ and $\frac{\partial \psi}{\partial N}$ are zero (the coordinates are independent), the equation of motion for the scalar quantum field $\varphi$ is

$$
\stackrel{\star \star}{\varphi}+3 \stackrel{\star}{\varphi}-e^{-2 N} \nabla_{r}^{2} \varphi-\left[4 \psi \frac{\partial \varphi}{\partial \psi}+\psi^{2} \frac{\partial^{2} \varphi}{\partial \psi^{2}}\right]=0,
$$

where the overstar denotes the derivative with respect to $N$ and $\varphi \equiv \varphi(N, \vec{r}, \psi)$. The commutator between $\varphi$ and $\Pi^{N}=\frac{\partial \mathcal{L}}{\partial \varphi, N}=g^{N N} \varphi_{, N}$ is given by

$$
\left[\varphi(N, \vec{r}, \psi), \Pi^{N}\left(N, \overrightarrow{r^{\prime}}, \psi^{\prime}\right)\right]=i g^{N N}\left|\frac{(5) g_{0}}{{ }^{(5)} g}\right| \delta^{(3)}\left(\vec{r}-\overrightarrow{r^{\prime}}\right) \delta\left(\psi-\psi^{\prime}\right),
$$

where $\left|\frac{(5) g_{0}}{{ }^{(5)} g}\right|$ is the inverse of the normalized volume of the manifold (1) and $g^{N N}=\psi^{-2}$. By means of the transformation $\varphi=\chi e^{-3 N / 2}\left(\frac{\psi_{0}}{\psi}\right)^{2}$ we obtain the 5D generalized Klein-Gordon like equation for $\chi(N, \vec{r}, \psi)$ and the commutator between $\chi$ and $\stackrel{\star}{\chi}$ :

$$
\begin{aligned}
& \stackrel{\star \star}{\chi}-\left[e^{-2 N} \nabla_{r}^{2}+\left(\psi^{2} \frac{\partial^{2}}{\partial \psi^{2}}+\frac{1}{4}\right)\right] \chi=0, \\
& {\left[\chi(N, \vec{r}, \psi), \stackrel{\star}{\chi}\left(N, \overrightarrow{r^{\prime}}, \psi^{\prime}\right)\right]=i \delta^{(3)}\left(\vec{r}-\overrightarrow{r^{\prime}}\right) \delta\left(\psi-\psi^{\prime}\right) .}
\end{aligned}
$$

The redefined field $\chi$ can be written in terms of a Fourier expansion in terms of the modes $\chi_{k_{r} k_{\psi}}(N, \vec{r}, \psi)=e^{i\left(\overrightarrow{k_{r}} \cdot \vec{r}+k_{\psi} \cdot \psi\right)} \xi_{k_{r} k_{\psi}}(N, \psi)$

$$
\begin{aligned}
\chi(N, \vec{r}, \psi) & =\frac{1}{(2 \pi)^{3 / 2}} \int d^{3} k_{r} \int d k_{\psi}\left[a_{k_{r} k_{\psi}} e^{i\left(\overrightarrow{k_{r}} \cdot \vec{r}+k_{\psi} \cdot \psi\right)} \xi_{k_{r} k_{\psi}}(N, \psi)\right. \\
& \left.+a_{k_{r} k_{\psi}}^{\dagger} e^{-i\left(\overrightarrow{k_{r}} \cdot \vec{r}+k_{\psi} \cdot \psi\right)} \xi_{k_{r} k_{\psi}}^{*}(N, \psi)\right],
\end{aligned}
$$

where the asterisk denotes the complex conjugate and $\left(a_{k_{r} k_{\psi}}, a_{k_{r} k_{\psi}}^{\dagger}\right)$ are respectively the annihilation and creation operators which satisfy the following commutation expressions

$$
\begin{aligned}
& {\left[a_{k_{r} k_{\psi}}, a_{k_{r}^{\prime} k_{\psi}^{\prime}}^{\dagger}\right]=\delta^{(3)}\left(\overrightarrow{k_{r}}-\overrightarrow{k_{r}^{\prime}}\right) \delta\left(\overrightarrow{k_{\psi}}-\overrightarrow{k_{\psi}^{\prime}}\right),} \\
& {\left[a_{k_{r} k_{\psi}}^{\dagger}, a_{k_{r}^{\prime} k_{\psi}^{\prime}}^{\dagger}\right]=\left[a_{k_{r} k_{\psi}}, a_{k_{r}^{\prime} k_{\psi}^{\prime}}\right]=0 .}
\end{aligned}
$$


The expression (7) complies if the modes are normalized by the following condition:

$$
\xi_{k_{r} k_{\psi}}\left(\stackrel{\star}{\xi}_{k_{r} k_{\psi}}\right)^{*}-\left(\xi_{k_{r} k_{\psi}}\right)^{*} \stackrel{\star}{\xi_{k_{r} k_{\psi}}}=i
$$

This equation provides the normalization for the complete set of solutions on all the $\left(k_{r}, k_{\psi}\right)$ spectrum. On the other hand, the dynamics for the modes $\xi_{k_{r} k_{\psi}}(N, \psi)$ is given by the equation

$$
\stackrel{\star \star}{\xi}_{k_{r} k_{\psi}}+k_{r}^{2} e^{-2 N} \xi_{k_{r} k_{\psi}}+\psi^{2}\left(k_{\psi}^{2}-2 i k_{\psi} \frac{\partial}{\partial \psi}-\frac{\partial^{2}}{\partial \psi^{2}}-\frac{1}{4 \psi^{2}}\right) \xi_{k_{r} k_{\psi}}=0 .
$$

The solution of (12) with the condition (11) can be written as [10]

$$
\xi_{k_{r} k_{\psi}}(N, \psi)=\frac{i \sqrt{\pi}}{2} e^{-i \vec{k}_{\psi} \cdot \vec{\psi}} \mathcal{H}_{1 / 2}^{(2)}\left[k_{r} e^{-N}\right]=e^{-i \vec{k}_{\psi} \cdot \vec{\psi}} \bar{\xi}_{k_{r}}(N)
$$

where $\mathcal{H}_{1 / 2}^{(1,2)}[x(N)]=\mathcal{J}_{1 / 2}[x(N)] \pm i \mathcal{Y}_{1 / 2}[x(N)]$ are the Hankel functions, $\mathcal{J}_{1 / 2}[x(N)]$ and $\mathcal{Y}_{1 / 2}[x(N)]$ are the first and second kind Bessel functions with $x(N)=k_{r} e^{-N}$. Furthermore the function $\bar{\xi}_{k_{r}}(N)$ is given by

$$
\bar{\xi}_{k_{r}}(N)=\frac{i \sqrt{\pi}}{2} \mathcal{H}_{1 / 2}^{(2)}\left[k_{r} e^{-N}\right]
$$

such that the normalization condition for $\bar{\xi}_{k_{r}}(N)$ becomes

$$
\bar{\xi}_{k_{r}}\left(\overline{\bar{\xi}}_{k_{r}}\right)^{*}-\left(\bar{\xi}_{k_{r}}\right)^{*} \stackrel{\star}{\bar{\xi}}_{k_{r}}=i
$$

Finally, the field $\chi$ in eq. (8) can be rewritten as

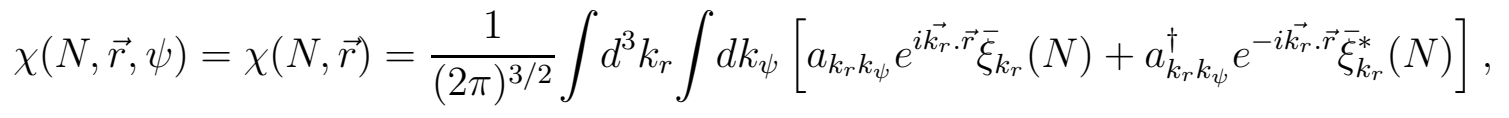

and the field $\varphi$ is given by

$$
\varphi(N, \vec{r}, \psi)=e^{-\frac{3 N}{2}}\left(\frac{\psi_{0}}{\psi}\right)^{2} \chi(N, \vec{r}),
$$

with $\chi(N, \vec{r})$ given by eq. (16). Note that exponentials $e^{ \pm i \vec{k}_{\psi} \cdot \vec{\psi}}$ disappear in $\chi(N, \vec{r})$ and there is not dependence on the fifth coordinate $\psi$ in this field. This is a very important fact that says us that the field $\varphi(N, \vec{r}, \psi)$ propagates only on the 3D spatially isotropic space $r(x, y, z)$, but not on the additional space-like coordinate $\psi$. Hence, gravity should not be localized on the fifth dimension and therefore the usual Newton law should not hold on the 5D manifold. 


\section{B. Coarse-granning of $\varphi$ in a $5 \mathrm{D}$ vacuum state}

To study the evolution of the field $\varphi$ on large 3D spatial scales, we can introduce the field $\chi_{L}$

$$
\chi_{L}(N, \vec{r})=\frac{1}{(2 \pi)^{3 / 2}} \int d^{3} k_{r} \int d k_{\psi} \Theta\left(\epsilon k_{0}(N)-k_{r}\right)\left[a_{k_{r} k_{\psi}} e^{i \overrightarrow{k_{r}} \cdot \vec{r}} \bar{\xi}_{k_{r}}(N)+c . c .\right],
$$

where $\Theta$ denotes the Heaviside function. Furthermore, c.c. denotes the complex conjugate of the first term inside the brackets and $k_{0}=1 / 2 e^{N}$ is the $N$-dependent wavenumber (related to the 3D spatially isotropic, homogeneous and flat space $r^{2}=x^{2}+y^{2}+z^{2}$ ), which separates the long $\left(k_{r}^{2} \ll k_{0}^{2}\right)$ and short $\left(k_{r}^{2} \gg k_{0}^{2}\right)$ wavelength sectors. Modes with $k_{r} / k_{0}<\epsilon$ are referred to as outside the horizon.

If the short wavelenght modes are described with the field $\chi_{S}$

$$
\chi_{S}(N, \vec{r})=\frac{1}{(2 \pi)^{3 / 2}} \int d^{3} k_{r} \int d k_{\psi} \Theta\left(k_{r}-\epsilon k_{0}(N)\right)\left[a_{k_{r} k_{\psi}} e^{i \overrightarrow{k_{r} \cdot \vec{r}}} \vec{\xi}_{k_{r}}(N)+\text { c.c. }\right],
$$

such that $\chi=\chi_{L}+\chi_{S}$, hence the equation of motion for $\chi_{L}$ will be approximately

$$
\stackrel{\star \star}{\chi}_{L}-\left(\frac{k_{0}(N) b_{0}}{b}\right)^{2} \chi_{L}=\epsilon\left[k_{0}^{\star \star} \eta(N, \vec{r})+k_{0}^{\star} \kappa(N, \vec{r})+2 \stackrel{\star}{k}_{0} \gamma(N, \vec{r})\right],
$$

where the stochastic operators $\eta, \kappa$ and $\gamma$ are given respectively by

$$
\begin{aligned}
& \eta(N, \vec{r})=\frac{1}{(2 \pi)^{3 / 2}} \int d^{3} k_{r} \int d k_{\psi} \delta\left(\epsilon k_{0}(N)-k_{r}\right)\left[a_{k_{r} k_{\psi}} e^{i \overrightarrow{k_{r} \cdot \vec{r}}} \bar{\xi}_{k_{r}}(N)+c . c .\right] \\
& \kappa(N, \vec{r})=\frac{1}{(2 \pi)^{3 / 2}} \int d^{3} k_{r} \int d k_{\psi} \stackrel{\star}{\delta}\left(\epsilon k_{0}(N)-k_{r}\right)\left[a_{k_{r} k_{\psi}} e^{i \overrightarrow{k_{r}} \cdot \vec{r}} \bar{\xi}_{k_{r}}(N)+c . c .\right] \\
& \gamma(N, \vec{r})=\frac{1}{(2 \pi)^{3 / 2}} \int d^{3} k_{r} \int d k_{\psi} \delta\left(\epsilon k_{0}(N)-k_{r}\right)\left[a_{k_{r} k_{\psi}} e^{i \overrightarrow{k_{r} . \vec{r}}} \stackrel{\star}{\xi}_{k_{r}}(N)+c . c .\right]
\end{aligned}
$$

The equation (20) can be rewritten as

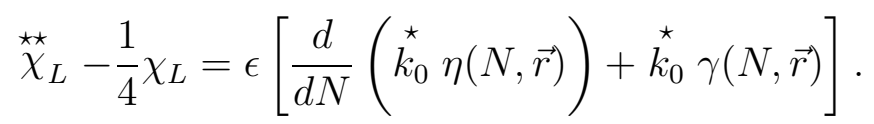

This is a Kramers-like stochastic equation that can be written as two first order stochastic (Langevin) ones by introducing the auxiliar field $u=\stackrel{\star}{\chi_{L}}-\epsilon \stackrel{\star}{k_{0}} \eta$

$$
\begin{aligned}
\stackrel{\star}{u} & =\frac{1}{4} \chi_{L}+\epsilon \stackrel{\star}{k_{0}} \gamma, \\
\stackrel{\star}{\chi}_{L} & =u+\epsilon \stackrel{\star}{k_{0}} \eta .
\end{aligned}
$$

The role of the noise $\gamma$ can be minimized in the system $(25)-(26)$ if $\left(k_{0}^{\star}\right)^{2}\left\langle\gamma^{2}\right\rangle \ll\left(\begin{array}{l}\star \star \\ k_{0}\end{array}\right)^{2}\left\langle\eta^{2}\right\rangle$, which holds when 


$$
\frac{\bar{\xi}_{k_{r}}^{\star}\left(\bar{\xi}_{k_{r}}\right)^{*}}{\bar{\xi}_{k_{r}}\left(\bar{\xi}_{k_{r}}\right)^{*}} \ll 1
$$

In that case the system $(25),(26)$ can be approximated to

$$
\begin{aligned}
\stackrel{\star}{u} & =\alpha \chi_{L}, \\
\stackrel{\star}{\chi}_{L} & =u+\epsilon \stackrel{\star}{k_{0}} \eta .
\end{aligned}
$$

This system represents two Langevin equations with a noise $\eta$ which is gaussian and white in nature

$$
\begin{aligned}
& \langle\eta\rangle=0 \\
& \left\langle\eta^{2}\right\rangle=\frac{\epsilon\left(k_{0}\right)^{2}}{2 \pi^{2} k_{0}^{\star}} \int d k_{\psi} \bar{\xi}_{\epsilon k_{0}} \bar{\xi}_{\epsilon k_{0}}^{*} \delta\left(N-N^{\prime}\right) .
\end{aligned}
$$

The equation that describes the dynamics of the transition probability $P\left[\chi_{L}^{(0)}, u^{(0)} \mid \chi_{L}, u\right]$ from a configuration $\left(\chi_{L}^{(0)}, u^{(0)}\right)$ to $\left(\chi_{L}, u\right)$ is a Fokker-Planck one

$$
\frac{\partial P}{\partial N}=-u \frac{\partial P}{\partial \chi_{L}}-\frac{1}{4} \chi_{L} \frac{\partial P}{\partial u}+\frac{1}{2} D_{11} \frac{\partial^{2} P}{\partial \chi_{L}^{2}},
$$

where $D_{11}=\frac{1}{2}\left(\epsilon \hat{k}_{0}^{\star}\right)^{2}\left[\int d N\left\langle\eta^{2}\right\rangle\right]$ is the diffusion coefficient related to the variable $\chi_{L}$. Explicitely

$$
D_{11}=\frac{\epsilon^{3}\left(k_{0}\right)^{2}}{4 \pi^{2}} k_{0}^{\star} \int d k_{\psi} \bar{\xi}_{\epsilon k_{0}} \bar{\xi}_{\epsilon k_{0}}^{*}
$$

which is divergent.

\section{The metric}

Now we consider the $5 \mathrm{D}$ canonical metric (1). In the $3 \mathrm{D}$ comoving frame $U^{r}=0$, the geodesic dynamics $\frac{d U^{C}}{d S}=-\Gamma_{A B}^{C} U^{A} U^{B}$ with $g_{A B} U^{A} U^{B}=1$, give us the velocities $U^{A}$ (latin letters take values $0,1,2,3,4$ )

$$
U^{N}=\frac{u(N)}{\psi \sqrt{u^{2}(N)-1}}, \quad U^{r}=0, \quad U^{\psi}=-\frac{1}{\sqrt{u^{2}(N)-1}},
$$

which are satisfied for $S(N)= \pm|N|$. In this work we shall consider the case $S(N)=|N|$. Note that the solution (34) is one of the possible representations of the general solution 


$$
\begin{aligned}
U^{N} & =\frac{\cosh [S(N)]}{\psi} \\
U^{r} & =0 \\
U^{\psi} & =-\sinh [S(N)] .
\end{aligned}
$$

In the representation (34) we obtain $\frac{d \psi}{d N} \equiv \frac{U^{\psi}}{U^{N}}=\psi / u(N)$, where $u(N)$ is an arbitrary function such that $\tanh [S(N)]=-1 / u(N)$. Thus, the fifth coordinate evolves as

$$
\psi(N)=\psi_{0} e^{\int d N / u(N)} .
$$

Here, $\psi_{0}$ is a constant of integration that has spatial units. From the mathematical point of view, we are taking a foliation of the 5D metric (1) with $r$ constant. Hence, to describe the metric in physical coordinates we must make the following transformations [9]:

$$
t=\int \psi(N) d N, \quad R=r \psi, \quad L=\psi(N) e^{-\int d N / u(N)},
$$

such that for $\psi(t)=1 / h(t)$, we obtain the $5 \mathrm{D}$ metric

$$
d S^{2}=\theta\left(d t^{2}-e^{2 \int h(t) d t} d R^{2}-d L^{2}\right)
$$

where $L=\psi_{0}$ is a constant and $h(t)=\dot{b} / b$ is the effective Hubble parameter and $b$ is the effective scale factor of the universe. The metric (40) describes a 5D generalized FRW metric, which is 3D spatially flat [i.e., it is flat in terms of $\vec{R}=(X, Y, Z)$ ], isotropic and homogeneous. In the representation $(\vec{R}, t, L)$, the velocities $\hat{U}^{A}=\frac{\partial \hat{x}^{A}}{\partial x^{B}} U^{B}$, are

$$
\hat{U}^{t}=\frac{2 u(t)}{\sqrt{u^{2}(t)-1}}, \quad \hat{U}^{R}=-\frac{2 R h}{\sqrt{u^{2}(t)-1}}, \quad \hat{U}^{L}=0
$$

where the old velocities $U^{B}$ are $U^{N}, U^{r}=0$ and $U^{\psi}$ and the velocities $\hat{U}^{B}$ are constrained by the condition

$$
\hat{g}_{A B} \hat{U}^{A} \hat{U}^{B}=1 .
$$

Note that the metric (40) is not globally flat and the line element (40) do not describes a 5D vacuum state. Furthermore, we are considering an observer which is in the frame described by the velocities (41). To avoid confusion, notice that the transformation (39) only describes a map from the particular frame (34) [of the metric (1)], to the particular frame (41) [of the metric (40)]. However, the transformation (39) is not a general map from the metric (1) to (40). Hence, if an observer is in the frame (41), he only see the effective 4D FRW metric

$$
d S^{2} \rightarrow d s^{2}=\theta\left(d t^{2}-e^{2 \int h(t) d t} d R^{2}\right)
$$

which has an effective $4 \mathrm{D}$ scalar curvature ${ }^{(4)} \mathcal{R}=6\left(\dot{h}+2 h^{2}\right)$. The metric (43) has a metric tensor with components $g_{\mu \nu}(\mu, \nu$ take the values $0,1,2,3)$. The absolute value of the determinant for this tensor is $\left|{ }^{(4)} g\right|=\left(b / b_{0}\right)^{6}$. Note that $\phi_{0}$ not necessarily takes the usual 4D Planckian length. Furthermore, the 4D energy density $\rho$ and the pressure p are 


$$
\begin{aligned}
& 8 \pi G \rho=3 h^{2}, \\
& 8 \pi G \mathrm{p}=-\left(3 h^{2}+2 \dot{h}\right),
\end{aligned}
$$

where $\dot{h}<0$ during all the evolution of the universe.

The function $u$ can be written as a function of the cosmic time $u(t)=-\frac{h^{2}}{\dot{h}}$, where the overdot represents the derivative with respect to $t$. The solution $N=\operatorname{arctanh}[1 / u(t)]$ corresponds to a time dependent power-law expanding universe $h(t)=p(t) t^{-1}$, such that the effective scale factor goes as $b \sim e^{\int p(t) / t d t}$. When $u^{2}(t)>1$, the velocities $\hat{U}^{t}$ and $\hat{U}^{R}$ are real, so that the condition (42) implies that $\theta=1$. [Note that the function $u(t)$ can be related to the deceleration parameter $\left.q(t)=-\ddot{b} b / \dot{b}^{2}: u(t)=1 /[1+q(t)].\right]$ In such a case the expansion of the universe is accelerated $(\ddot{b}>0)$. However, when $u^{2}<1$ the velocities $U^{t}$ and $U^{R}$ are imaginary and the condition (42) holds for $\theta=-1$. In this case the expansion of the universe is decelerated because $\ddot{b}<0$. So, the parameter $\theta$ is introduced in the metric (40) to preserve the hyperbolic condition (42). The new coordinate $R$ gives us the physical distance between galaxies separated by cosmological distances: $R(t)=r(t) / h(t)$, where $r(t)$ is given by $3 u^{2}(t)=4 r^{2}(t)\left(b / b_{0}\right)^{2}-1$ :

$$
r^{2}(t)=\left[\frac{3}{4} \frac{h^{4}}{\dot{h}^{2}}+\frac{1}{4}\right] e^{-2 \int h(t) d t},
$$

for a given evolution of the universe described with $b(t)$. For $r>1(r<1)$, the 3D spatial distance $R(t)$ is defined on super (sub) Hubble scales.

\section{The inflaton field in an effective 4D FRW metric}

The 4D Lagrangian corresponding to the effective 4D Friedmann-Robertson-Walker (FRW) metric (43) [i.e., in the frame (41)], is given by

$$
{ }^{(4)} \mathcal{L}\left(\varphi, \varphi_{, \mu}\right)=-\sqrt{\left|\frac{{ }^{(4)} g}{{ }^{(4)} g_{0}}\right|}\left[\frac{1}{2} g^{\mu \nu} \varphi_{, \mu} \varphi_{, \nu}+V(\varphi)\right],
$$

where the effective potential for the 4D FRW metric (43), is

$$
V(\varphi)=-\left.\frac{1}{2} g^{\psi \psi} \varphi_{, \psi} \varphi_{, \psi}\right|_{\psi=h^{-1}}=\left.\frac{1}{2}\left(\frac{\partial \varphi}{\partial \psi}\right)^{2}\right|_{\psi=h^{-1}} .
$$

In our case this potential in the frame $(41)$ and $\psi=1 / h$, takes the form

$$
V(\varphi)=2 h^{2}(t) \varphi^{2}(t, \vec{R}, L) .
$$

Notice this potential has a geometrical origin and takes different representations in different frames. In our case the observer is in a frame $\hat{U}^{L}=0$, because we are taking a foliation $L=\psi_{0}$ on the 5D metric (40). Furthermore, the effective 4D equation of motion for $\varphi$ is

$$
\ddot{\varphi}+\left(3 h-\frac{\dot{h}}{h}\right) \dot{\varphi}-e^{-2 \int h(t) d t} \nabla_{R}^{2} \varphi-\left.\left[\frac{4}{\psi} \frac{\partial \varphi}{\partial \psi}+\frac{\partial^{2} \varphi}{\partial \psi^{2}}\right]\right|_{\psi=h^{-1}}=0
$$


which means that the effective $4 \mathrm{D}$ expression for $\frac{d V(\varphi)}{d \varphi}$ is

$$
\left.V^{\prime}(\varphi)\right|_{\psi=h^{-1}}=2 h^{2}(t) \varphi(\vec{R}, t, L)-\frac{\dot{h}}{h} \dot{\varphi}(\vec{R}, t, L) .
$$

In order to simplify the structure of the equation (50) we can make the following transformation:

$$
\varphi(\vec{R}, t)=e^{-\frac{1}{2} \int(3 h-\dot{h} / h) d t} \chi(\vec{R}, t),
$$

such that we obtain the following $4 \mathrm{D}$ Klein-Gordon equation for $\chi$

$$
\ddot{\chi}-\left[e^{-2 \int h(t) d t} \nabla_{R}^{2}+\frac{h^{2}}{4}+\frac{3}{4}\left(\frac{\dot{h}}{h}\right)^{2}-\frac{1}{2} \frac{\ddot{h}}{h}\right] \chi=0 .
$$

The field $\chi$ can be expanded as a Fourier's representation in terms of the modes $\chi_{k_{R} k_{L}}(\vec{R}, t)=$ $e^{i \overrightarrow{k_{R}} \cdot \vec{R}} \bar{\xi}_{k_{R}}(t)$

$$
\chi(\vec{R}, t)=\frac{1}{(2 \pi)^{3 / 2}} \int d^{3} k_{R} \int d k_{L}\left[a_{k_{R} k_{L}} e^{i \overrightarrow{k_{R}} \cdot \vec{R}} \bar{\xi}_{k_{R}}(t)+c . c .\right] \delta\left(k_{L}-k_{\psi_{0}}\right),
$$

where the dynamics for the modes $\bar{\xi}_{k_{R}}(t)$ is given by

$$
\ddot{\bar{\xi}}_{k_{R}}+\left[k_{R}^{2} e^{-2 \int h(t) d t}-\frac{h^{2}}{4}-\frac{3}{4}\left(\frac{\dot{h}}{h}\right)^{2}+\frac{1}{2} \frac{\ddot{h}}{h}\right] \bar{\xi}_{k_{R}}=0 .
$$

It is important to notice that eq. (55) is exactly the equation for $\bar{\xi}_{k_{r}}(N)$ with the variables transformation (39), on the hypersurface $\psi=h^{-1}$.

\section{E. Coarse-granning of $\varphi$ in an effective $4 \mathrm{D}$ cosmological metric}

Now we can define the fields $\chi_{L}(t, \vec{R})$ and $\chi_{S}(t, \vec{R})$, which describe respectively the long and short wavelength sectors of the field $\chi$ on the effective 4D FRW metric (43)

$$
\begin{aligned}
& \chi_{L}(t, \vec{R})=\frac{1}{(2 \pi)^{3 / 2}} \int d^{3} k_{R} \int d k_{L} \Theta\left(\epsilon F(t)-k_{R}\right)\left[a_{k_{R} k_{\psi}} e^{i \overrightarrow{k_{R}} \cdot \vec{R}} \bar{\xi}_{k_{R}}(t)+c . c .\right] \delta\left(k_{L}-k_{\psi_{0}}\right), \\
& \chi_{S}(t, \vec{R})=\frac{1}{(2 \pi)^{3 / 2}} \int d^{3} k_{R} \int d k_{L} \Theta\left(k_{R}-\epsilon F(t)\right)\left[a_{k_{R} k_{\psi}} e^{i \overrightarrow{k_{R}} \cdot \vec{R}} \bar{\xi}_{k_{R}}(t)+c . c .\right] \delta\left(k_{L}-k_{\psi_{0}}\right),
\end{aligned}
$$

where $F(t)=h(t) e^{\int h d t}$ is the inverse of the Hubble horizon in an expanding universe. The field that describes the dynamics of $\chi$ on the infrared sector $\left(k_{R}<\epsilon F\right)$ is $\chi_{L}$. During the inflationary expansion the dimensionless parameter take values of the order of $10^{-3}-10^{-4}$. However, the present day value for $\epsilon$ should be of the order of $10^{2}$. The dynamics of $\chi_{L}$ obeys the Kramers-like stochastic equation 


$$
\ddot{\chi}_{L}-\frac{k_{0}^{2} b_{0}^{2}}{b^{2}} \chi_{L}=\epsilon\left[\frac{d}{d t}(\dot{F} \eta(t, \vec{R}))+\dot{F} \gamma(t, \vec{R})\right],
$$

where $k_{0}(t)=e^{\int h d t}\left[\frac{h^{2}}{4}+\frac{3}{4}(\dot{h} / h)^{2}-\frac{1}{2} \ddot{h} / h\right]^{1 / 2}$ and the stochastic operators $\eta, \kappa$ and $\gamma$ are

$$
\begin{aligned}
& \eta=\frac{1}{(2 \pi)^{3 / 2}} \int d^{3} k_{R} \delta\left(\epsilon F-k_{R}\right)\left[a_{k_{R} k_{\psi_{0}}} e^{i \overrightarrow{k_{R}} \cdot \vec{R}} \bar{\xi}_{k_{R}}(t)+c . c .\right] \\
& \gamma=\frac{1}{(2 \pi)^{3 / 2}} \int d^{3} k_{R} \delta\left(\epsilon F-k_{R}\right)\left[a_{k_{R} k_{\psi_{0}}} e^{i \overrightarrow{k_{R}} \cdot \vec{R}} \dot{\bar{\xi}}_{k_{R}}(t)+c . c .\right] .
\end{aligned}
$$

This second order stochastic equation can be rewritten as two Langevin stochastic equations

$$
\begin{aligned}
& \dot{u}=\frac{k_{0}^{2} b_{0}^{2}}{b^{2}} \chi_{L}+\epsilon \dot{F} \gamma \\
& \dot{\chi}_{L}=u+\epsilon \dot{F} \eta
\end{aligned}
$$

where $u=\dot{\chi}_{L}-\epsilon \dot{F} \gamma$. The condition to neglect the noise $\gamma$ with respect to $\eta$, now holds

$$
\frac{\dot{\bar{\xi}}_{k_{R}} \dot{\bar{\xi}}_{k_{R}}}{\bar{\xi}_{k_{R}} \bar{\xi}_{k_{R}}^{*}} \ll \frac{(\ddot{F})^{2}}{(\dot{F})^{2}},
$$

Notice this result is exactly the same in eq. (27), with the transformation (39). The FokkerPlanck equation for the transition probability $\bar{P}\left(\chi_{L}^{(0)}, u^{(0)} \mid \chi_{L}, u\right)$ is

$$
\frac{\partial \bar{P}}{\partial t}=-u \frac{\partial \bar{P}}{\partial \chi_{L}}-\frac{k_{0}^{2} b_{0}^{2}}{b^{2}} \chi_{L} \frac{\partial \bar{P}}{\partial u}+D_{11}(t) \frac{\partial^{2} \bar{P}}{\partial \chi_{L}^{2}}
$$

where $\bar{D}_{11}(t)=\frac{\epsilon^{3} \dot{F} k_{0}^{2}}{4 \pi^{2}}\left|\bar{\xi}_{\epsilon F}\right|^{2}$. Hence, the equation of motion for $\left\langle\chi_{L}^{2}\right\rangle=\int d \chi_{L} d u \chi_{L}^{2} \bar{P}\left(\chi_{L}, u\right)$ is

$$
\frac{d}{d t}\left\langle\chi_{L}^{2}\right\rangle=\bar{D}_{11}(t)
$$

When $\bar{D}_{11}>0, \chi_{L}$ increases its number of degrees of freedom. In our case the number of degrees of freedom changes depending on the rate of expansion of the universe. In order to return to the original field $\varphi_{L}=e^{-\frac{1}{2} \int\left(3 h-\frac{h}{h}\right)} \chi_{L}$ the equation (65) can be rewritten as

$$
\frac{d}{d t}\left\langle\varphi_{L}^{2}\right\rangle=-\left(3 h-\frac{\dot{h}}{h}\right)\left\langle\varphi_{L}^{2}\right\rangle+\bar{D}_{11}(t) e^{-\int\left(3 h-\frac{\dot{h}}{h}\right) d t}
$$

which has the following general solution

$$
\left\langle\varphi_{L}^{2}\right\rangle=e^{-\int^{t}\left(3 h-\frac{\dot{h}}{h}\right) d t^{\prime \prime}}\left[\int^{t} \bar{D}_{11}\left(t^{\prime}\right) d t^{\prime}+C\right],
$$

where $C$ is a constant of integration, $\bar{\xi}_{k_{R}=\epsilon F}$ is the solution of eq. (55) with $k_{R}=\epsilon F$. 


\section{EVOLUTION OF THE UNIVERSE: A MODEL}

With the aim to study an effective model for the expansion of the universe we consider a Hubble parameter $h(t)=p(t) / t$, such that the time dependent power expansion $p(t)$ is given by

$$
p(t)=1.8 a t^{-n}-1.8 b t^{-n / 2}+\left(\frac{b^{2}}{4 a}+\frac{2}{3}\right)+c t,
$$

where $a=1 / 610^{30 n} G^{n / 2}, b=1 / 310^{15 n} G^{n / 4}, c=10^{-61} G^{-1 / 2}$ and $n=0.352$. This model represents an early inflationary expansion followed by a decelerated (radiation dominated followed by a matter dominated expansion) that finally suffers a present day quintessential accelerated expansion. Other cosmological models without inflationary expansion has been considered recently in the literature [11]. The power of expansion (68) is shown in the figure $(1)$, being $x(t)=\log _{10}\left[t / t_{0}\right]$. Note that for $x<10, p(t)$ suffers an inflationary expansion. For $60.135>x>10$, the universe is decelerated, being radiation dominated for $x \simeq 30$ and matter dominated for $x \simeq 55$. For $x>60.135$ the universe suffers a quintessential expansion, being its actual age $x \simeq 60.653$ (i.e., approximately $t \simeq 1.510^{10}$ years old), which has been experimentally observed from the supernova (SNe) Type data [12-15]. The model here studied gives us a present day deceleration parameter $q=-\ddot{b} b / \dot{b}^{2}: q(x=60.653) \simeq-0.492$, which is in good agreement with experimentation [16]. The figure (2) shows the function $r(t)$, which decreases monotonically. The function $r(t)=\frac{\lambda_{\text {phys }}}{\lambda_{H}}$ [given by eq. (46)], describes the evolution of the physical wavelength $\lambda_{\text {phys }}$ relative to the horizon wavelength $\lambda_{H}=1 / h$. Note that $r(t)$ remains below the unity for $x>0.001$. Hence, relativistic causality only should be violated on transplanckian temporal scales (i.e., for $x<0.001$ ). The origin of this partial violation should be of quantum mechanical nature.

\section{A. Estimation of $\left\langle\varphi_{L}^{2}\right\rangle$}

The equation (55) is very difficult to solve for a time dependent power-law as (68). Hence, to make an estimation of $\left\langle\varphi_{L}^{2}\right\rangle$ for different stages of the evolution of the universe we shall consider that $p$ is nearly constant (i.e., $\frac{\dot{p}}{p} \ll p / t$ ). By solving the eq. (55), we obtain

$$
\bar{\xi}_{k_{R}}[y(t)]=i \sqrt{\frac{\pi}{4(p-1)}} \sqrt{\frac{t}{t_{0}}} \mathcal{H}_{\nu}^{(2)}[y(t)]
$$

where $y(t)=\frac{k_{r} t_{0}^{p} t^{1-p}}{p-1}, \nu=\frac{p}{2(p-1)}, \mathcal{H}_{\nu}^{(2)}$ is the second kind Hankel function and $t_{0}$ corresponds to time when inflation begins. In order to obtain $\left\langle\varphi_{L}^{2}\right\rangle$ from eq. (67), it is necessary to find $\bar{D}_{11}$, which is given by

$$
\bar{D}_{11}(t)=\frac{\epsilon^{3} \dot{F} k_{0}^{2}}{4 \pi^{2}}\left|\bar{\xi}_{\epsilon F}\right|^{2} \simeq \frac{\epsilon^{2}}{16 \pi^{2}} \frac{(p-1)\left(p^{2}-1\right)}{t_{0}^{3 p+1}} t^{2 p-1},
$$

where we have made use of the fact that $k_{0}^{2}(t)=\frac{t^{2(p-1)}}{4 t_{0}^{2 p}}\left(p^{2}-1\right)($ which is negative for $p<1)$, and the small argument asymptotic expansion for the second kind Hankel function. Under this approximation, the expectation value for the second momenta of $\varphi_{L}$ is 


$$
\left\langle\varphi_{L}^{2}\right\rangle \simeq\left[\frac{\epsilon^{2}}{16 \pi^{2}} \frac{(p-1)\left(p^{2}-1\right)}{2 p t_{0}^{3 p+1}} t^{2 p}+C\right]\left(\frac{t}{t_{0}}\right)^{-(3 p+1)}
$$

where $C$ is an arbitrary constant of integration. Notice that for $p=3 / 2$ it has a singularity. One interesting case is $p=1 / 2$, that corresponds to the radiation dominated universe. In this case $\left\langle\varphi_{L}^{2}\right\rangle$ is given by

$$
\left\langle\varphi_{L}^{2}\right\rangle \simeq\left[\frac{3 \epsilon^{2}}{2^{7} t_{0}^{5 / 2}} t+C\right]\left(\frac{t}{t_{0}}\right)^{-5 / 2}
$$

Notice that $\left\langle\varphi_{L}^{2}\right\rangle$ decreases with the time for all values of $p$. It is important to note that this approach is only valid when the condition (63) is fulfilled. Note that $k_{0}^{2}>0$ for $p>1$ and $k_{0}^{2}<0$ for $p<1$. However $\dot{F}>0$ for $p>1$ and $\dot{F}<0$ for $p<1$, so that $\bar{D}_{11}>0$, for all values of $p$. This means that the number of degrees of freedom for $\chi_{L}$ is always increasing. However the relevant field for us is $\varphi_{L}$. Note that its number of degrees of freedom is always increasing but its rate of increment is now decreasing because of the present day (and future) acceleration of the universe. Hence, the model predicts a decreasing rate in the increment of the number of degrees of freedom, which (for $t \rightarrow \infty$ ) will be almost constant. Taking $p$ almost constant from (63) we obtain that

$$
\frac{1}{4} \ll(p-2)^{2}
$$

This means that the stochastic approach here developed is very efficient for $p \gg 2$ and has a reasonably good behavior for $p<1$ (we are dealing only with $p>0$ ). However, for $p \simeq 2$, the stochastic noise $\gamma$ in eq. (61) should be taken into account.

\section{B. Calculation of the background field $\phi_{c}(t)$}

In order to estimate the evolution of the squared inflaton fluctuations in different epochs of the evolution of the universe, we can make a semiclassical approach $\varphi(t, \vec{R})=\phi_{c}(t)+$ $\phi(t, \vec{R})$, such that $\phi_{c}(t)=\langle\varphi\rangle$ and $\langle\phi\rangle=0$. Hence,

$$
\left\langle\varphi^{2}\right\rangle=\phi_{c}^{2}(t)+\left\langle\phi^{2}\right\rangle \text {. }
$$

To estimate $\left\langle\phi^{2}\right\rangle$, we need to know $\phi_{c}(t)$, which is the zero mode solution of the differential equation (50) and has the general solution

$$
\phi_{c}(t)=e^{-\int p(t) / t d t}\left(A e^{-\int p(t) / t d t}+B\right)
$$

where $A$ and $B$ are constants of integration. For the time dependent power-law (68), the solution is

$$
\phi_{c}(t)=t^{-\left(\frac{b^{2}}{4 a}+\frac{2}{3}\right)} e^{1.8\left(\frac{a}{n}\right) t^{-n}-3.6\left(\frac{b}{n}\right) t^{-n / 2}-c t}\left[A t^{-\left(\frac{b^{2}}{4 a}+\frac{2}{3}\right)} e^{1.8\left(\frac{a}{n}\right) t^{-n}-3.6\left(\frac{b}{n}\right) t^{-n / 2}-c t}+B\right] .
$$

Note that $\phi_{c}(t)$ decreases monotonically during all the history of the universe. 


\section{Estimation of squared inflaton fluctuations on the infrared sector}

Once known $\phi_{c}$, which becomes negligible for late times, we can estimate the squared inflaton fluctuations $\left\langle\phi_{L}^{2}\right\rangle$ on the infrared sector. For late times one obtains $\left\langle\varphi_{L}^{2}\right\rangle_{t \gg G^{1 / 2}} \simeq$ $\left\langle\phi_{L}^{2}\right\rangle_{t \gg G^{1 / 2}}$, where

$$
\left\langle\phi_{L}^{2}\right\rangle_{t \gg G^{1 / 2}} \simeq\left[\frac{\epsilon^{2}}{16 \pi^{2}} \frac{(p-1)\left(p^{2}-1\right)}{2 p t_{0}^{3 p+1}} t^{2 p}+C\right]\left(\frac{t}{t_{0}}\right)^{-(3 p+1)} .
$$

Notice that the expectation value for the inflaton field fluctuations decreases in all the epochs of the expansion of the universe, independently of the $C$-value.

\section{FINAL COMMENTS}

In this paper we have studied a nonperturbative scalar field governed cosmological model from a noncompact Kaluza-Klein theory of gravity from a 5D apparent vacuum. This vacuum is defined as a purely kinetic density Lagrangian for a scalar field minimally coupled to gravity on a $5 \mathrm{D}$ background globally flat $\left(R_{B C D}^{A}=0\right)$ metric. We have worked a cosmological model with a time dependent power-law $p(t)$ which describes an universe that initially is accelerated and suffers an inflationary expansion $(p>>1)$. However, this power decreases with time through its minimal value $p \simeq 1 / 2$, which describes a radiation dominated universe. Thereafter, this power begins to increase passing by a matter dominated $(p \simeq 2 / 3)$ epoch, to the present day quintessential expansion with $p \simeq 1.2$. The model predicts that $p$ will continue increasing and finally will expand with $p \simeq c t$. This means that finally the universe will adopt a de Sitter expansion. At this moment, the field $\phi_{c}(t)$ will tend to zero, when the system will adopt its minimum energetic configuration.

We agree with the suggestion that perhaps some scalar (inflaton field) has been sliding down its potential energy hill on a time scale of billions of years rather than fractions of second (i.e., only during inflation) $[17,8,10,18]$. We obtain that the mass of the inflaton field is of the order of the Hubble parameter and the scalar potential is quadratic in $\varphi$. Notice that this potential [see eq. (49)] is induced geometrically and take different representations in different frames. In our case, we are dealing with a frame which is comoving with the expansion of the universe. In agreement with the power-law (68), in the future the universe will be again dominated by vacuum $\mathrm{p} \simeq-\rho$, to die in a de Sitter (inflationary) expansion. The stochastic approach here developed has a good behavior for $p \gg 2$ and $p<1$, but not for $p \simeq 2$. In that case, a more complete stochastic formalism should be developed, in order to include the stochastic noise $\gamma$ in the evolution of $\left\langle\varphi_{L}^{2}\right\rangle$.

Finally, in our cosmlogical model the only free parameter is a cosmological observable; the Hubble parameter $h$. In this work we are dealing with some example given by eq. (68), but in general, the theory can make predictions from observation for the reconstruction of the Hubble parameter, which is expected from the future SNAP data [19].

\section{Acknowledgements}

MA and MB acknowledge CONICET (Argentina) and UNMdP for financial support. JEMA acknowledges CONACyT (México) and IFM for financial support. 


\section{REFERENCES}

[1] P. Forgacs and Z. Horvath, Gen. Rel. Grav. 11, 205 (1979); A. Chodos and S. Detweiler, Phys. Rev. D21, 2167 (1980); P. G. O. Freund, Nucl. Phys. B209, 146 (1982); T. Appelquist and A. Chodos, Phys. Rev. Lett. 50, 141 (1983); E. Alvarez and M. B. Gavela, Phys. Rev. Lett. 51, 931 (1983); S. Randjbar-Daemi, A. Salam and J. Strathdee, Phys. Lett. B135, 388 (1984).

[2] W. J. Marciano, Phys. Rev. Lett. 52, 489 (1984).

[3] L. Randall and R. Sundrum, Phys. Rev. Lett. 83, 4690 (1999); L. Randall and R. Sundrum, Phys. Rev. Lett. 83, 3370 (1999).

[4] N. Arkani-Hamed, S. Dimopoulos and G. R. Dvali, Phys. Lett. B429, 263 (1998).

[5] N. Arkani-Hamed, S. Dimopoulos and G. R. Dvali, Phys. Rev. D59, 086004 (1999).

[6] see, for example, J. M. Overdiun and P. S. Wesson, Phys. Rep. 283, 303 (1997), and references therein.

[7] J. W. van Holten, Phys. Rev. Lett. 89, 201301 (2002); J. W. van Holten, Mod. Phys. Lett. A17, 1383 (2002).

[8] J. E. Madriz Aguilar and M. Bellini, Eur. Phys. J. C38, 367 (2004).

[9] J. E. Madriz Aguilar, M. Bellini, Eur. Phys. J. C38, 123 (2004); Phys. Lett. B596, 116 (2004); Eur. Phys. J. C38, 367 (2004); J. E. Madriz Aguilar, M. Bellini, Extra force and extra mass from noncompact Kaluza-Klein theory in a cosmological model. E-print: gr-qc/0408054. To appear in Eur. Phys. J. C (2005).

[10] A. Raya Montaño, J. E. Madriz Aguilar and M. Bellini, A nonperturbative scalar field governed expansion of the universe from a noncompact Kaluza-Klein theory. E-print: gr-qc/0502027.

[11] H. Liu, Phys. Lett. B560, 149 (2003).

[12] A. G. Riess et al., Astron. J116, 1009 (1998).

[13] S. J. perlmutter et al., Astroph. J517, 565 (1999).

[14] R. A. Knop et al., Astroph. J598, 102(K) (2003).

[15] A. G. Riess et al., Astroph. J607, 665(R) (2004).

[16] Review of Particle Physics, Phys. Rev. D66, 010001-170 (2002).

[17] R. P. Kirshner, Science 300, 1914 (2003).

[18] A. Guth and D. Kaiser, Science 307, 884 (2005).

[19] A. Shafieloo, U. Alam, V. Sahni and A. Starobinsky, Smoothing Supernova Data to Reconstruct the Expansion History of the Universe.E-print: astro-ph/0505329. 
Fig.1: Evolution of $p[x(t)]$ as a function of $x(t)=\log _{10}(t)$.

Fig.2: Evolution of $r[x(t)]$ as a function of $x(t)=\log _{10}(t)$, during the early stages of inflation. 